\title{
Pencil and paper electrodes for pharmaceutical analyses
}

\author{
Volume 4 Issue I - 2017 \\ Isaac Yves Lopes de Mac do, Eric de Souza
Gil \\ Faculdade de Farmácia, Universidade Federal de Goiás, Brazil
}

Correspondence: Eric de Souza Gil, Faculdade de Farmácia, Universidade Federal de Goiás, Goiânia, GO, Brazil, Email ericsgil@gmail.com

Received: January 27, 2017| Published: January 30, 2017 non-widespread used electrodes e.g. paper-based, graphite pencil and imprinted electrodes can be efficient tools to analyze several products, i.e., foodstuffs, pharmaceuticals, environmental samples and different chemicals. The main appeal is concerned to the commercial accessibility at reduced prices. Moreover, if they are not promptly ready to use as an analytical tool, the easiness of their production overcomes such issue. It is outstanding that many studies have been carried out in order to improve and highlight the advantages of these economic devices. In turn, the most challenging drawback is related to the low homogeneity of their microstructure, which leads to oscillation of electroactive surface area, and thus low analytical reproducibility. Therefore, a number of biological or chemical modifiers upsurge as a promising solution to ally the analytical performance to financial aspects. In fact, both are mandatory demands of chemical analyses.

The conventional working electrodes (e.g. glassy carbon, carbon paste, boron-doped diamond) are commonly employed in lab analyses and also as standard parameter in researches focusing the electrochemistry in general. On the other hand, non-conventional i.e., paper-based, graphite pencil and imprinted electrodes are far less widespread devices, that can be modified in many ways, thus resulting in numerous cheaper alternatives, that can solve different analytical demands. For instance, paper-based electrodes and imprinted electrodes, besides the low cost, allow the design of miniaturized and sensing systems. ${ }^{1,2}$

The graphite pencil, either crude or modified, has been proved as valuable working electrodes for several electroanalytical applications. ${ }^{3}$ In addition to its cheapness, the soft surface of the pencil is easily removed through polishment in paper, which admits a practical and faster cleaning. Hence, owing to the electrode surface renewal, the measurements gain repeatability. ${ }^{4,5}$ The graphite structure is characterized by uneven graphite/clay distribution and considerable inner porosity, which diminishes its conductivity.

However, glassy carbon possesses higher conducting properties, surrounding better resolution and sensitivity, and better microstructural homogeneity that lead to higher reproducibility; there are an abysmal difference of prices. Therefore, taking into account the good analytical performance of pencil electrodes especially for samples, in which the quantity is not an issue, such alternative electrode may be a good choice.

Paper-based electrochemical devices also present similar cost/ benefit relationship. Indeed, the uniform graphite deposition on paper surface is a difficult task. Nevertheless the massive production of several devices with insignificant or almost immaterial amounts of biomass, set the paper based electrodes as the most inexpensive and eco-friendly alternatives.
Moreover the optimization of screen-printed technologies must overcome the difficulties regarding the standardization of electronic material deposition at paper surface, which certainly in a short time will solve the problems related to the lack of reproducibility.

In conclusion, paper and pencil alternative electrodes offer a viable opportunity to reduce cost and time of electroanalyses. Furthermore, the advances of such material science, may soon allow, the development of cheap and suitable devices for pharmaceutical analyses.

\section{Acknowledgments}

None.

\section{Conflicts of interest}

Author declares there are nno conflicts of interest.

\section{Funding}

None.

\section{References}

1. Dossi N, Toniolo R, Impellizzieri F, et al. Doped pencil leads for drawing modified electrodes on paper-based electrochemical devices. Journal of Electroanalytical Chemistry. 2014;722-723:90-94.

2. Mohamed HM. Screen-printed disposable electrodes: Pharmaceutical applications and recent developments. TrAC - Trends in Analytical Chemistry. 2016; 82: 1-11.

3. Abdul AM, Kawde AN. Gold nanoparticle-modified graphite pencil electrode for the high-sensitivity detection of hydrazine. Talanta. 2013;115:214-221. 
4. Dogan TB, Ozkan SA. Electrochemical determination of anticancer drug fulvestrant at dsDNA modified pencil graphite electrode. Electrochimica Acta. 2011;56(12):4433-4438.
5. Heydari H, Gholivand MB, Abdolmaleki A. Cyclic voltammetry deposition of copper nanostructure on MWCNTs modified pencil graphite electrode: An ultra-sensitive hydrazine sensor. Materials Science and Engineering: C. 2016;66:16-24 